\title{
Expression of Nuclear Factor Erythroid 2 Protein in Malignant Cutaneous Tumors
}

\author{
Chang Yong Choi ${ }^{1}$, Jin Young Kim ${ }^{1}$, Seo Yeong $\mathrm{Wee}^{1}$, Jang Hyun Lee ${ }^{2}$, Doo Hyun Nam³ \\ Chul Han $\mathrm{Kim}^{4}$, Moon Kyun $\mathrm{Cho}^{5}$, Yoon Jin Lee ${ }^{5}$, Hae Seon $\mathrm{Nam}^{5}$, Sang Han Lee, \\ Sung Woo Cho ${ }^{5}$ \\ ${ }^{1}$ Department of Plastic and Reconstructive Surgery, Soonchunhyang Gumi Hospital, Soonchunhyang University College of Medicine, Gumi; \\ ${ }^{2}$ Department of Plastic and Reconstructive Surgery, Hanyang University Guri Hospital, Hanyang University College of Medicine, Guri; \\ ${ }^{3}$ Department of Plastic and Reconstructive Surgery, Soonchunhyang University Cheonan Hospital, Cheonan; ${ }^{4}$ Department of Plastic and \\ Reconstructive Surgery, Soonchunhyang University College of Medicine, Seoul; ${ }^{5}$ Molecular Cancer Research, Soonchunhyang University \\ College of Medicine, Cheonan, Korea
}

Background Reactive oxygen species (ROS) damages cell molecules, and modifies cell signaling. The nuclear factor E2-related factor (Nrf2) is a critical transcription regulator, which protects cells against oxidative damage. Nrf2 expression is increased in a large number of cancers. However, little information has been reported regarding the expression of Nrf2 in skin cancers. Hence, we explored the expression of Nrf2 protein in skin cancers.

Methods The Nrf2 protein expression in 24 specimens, including 6 malignant melanomas (MM), 6 squamous cell carcinomas (SCC), 6 basal cell carcinomas (BCC), and 6 normal skin tissues, was evaluated by western blotting. Immunohistochemical staining was performed. The expression of Kelch-like ECH-associated protein 1 (Keap1), the key regulator of Nrf2, was also analyzed by western blotting.

Results Small interfering RNA transfection to the melanoma cell line G361 confirmed that an approximately $66 \mathrm{kDa}$ band was the true Nrf2 band. The western blot revealed that the Nrf2 protein was definitely expressed in normal skin tissues, but the Nrf2 expression was decreased in MM, SCC, and BCC. Immunohistochemical examination showed that expression of Nrf2 was decreased in all skin cancer tissues compared to the normal skin tissues. Keap1 was not expressed in all malignant skin tumors and normal skin tissues by western blot.

Conclusions ROS was increased in various types of cancers which proteins were highly expressed or underexpressed. This study demonstrated that the expression of Nrf2 protein was down-regulated in human malignant skin tumors. We suggest that decreased expression of Nrf2 is related to skin cancers.

Keywords NF-E2-related factor 2 / Reactive oxygen species / Skin neoplasms
Correspondence: Chul Han Kim Department of Plastic and Reconstructive Surgery, Soonchunhyang University College of Medicine, 59 Daesagwan-ro, Yongsan-gu, Seoul 140-743, Korea Tel: +82-2-709-9283

Fax: +82-2-796-3543

E-mail: kchann@hanmail.net

This work was supported by the Soonchunhyang University Research Fund.

No potential conflict of interest relevant to this article was reported.

Received: 3 Jan 2014 • Revised: 12 Feb 2014 • Accepted: 12 Feb 2014

pISSN: 2234-6163 • elSSN: 2234-6171 • http://dx.doi.org/10.5999/aps.2014.41.6.654 • Arch Plast Surg 2014;41:654-660

\section{INTRODUCTION}

Human skin is a major target of oxidative stress because it is fre- quently exposed to toxic environments such as ultraviolet light, chemicals, and ionizing radiation [1]. Oxidative stress disturbs the normal redox balance and results in the production of reac- 
tive oxygen species (ROS). ROS causes damage to the cells and modifies signal transduction. ROS causes pathological conditions such as cancer, arteriosclerosis, neurodegenerative diseases, and inflammation $[2,3]$. Therefore, for detoxification of ROS, cells use various strategies, such as generation of antioxidants and expression of ROS-detoxifying enzymes.

The nuclear factor related factor (Nrfs) family plays an important role in protecting the cells against oxidative damage. The Nrf gene consists of three components, Nrf1, Nrf2, and Nrf3. Among them, the nuclear factor E2-related factor (Nrf2) is involved in important skin cell pathways for protecting cells against oxidative stress, and it is also involved in skin adaptation to microenvironmental stress [4]. Nrf2 is primarily regulated by Kelch-like ECH-associated protein 1 (Keap1) [5]. Keap1 is a potent cytosolic repressor of Nrf2, and Keap1 has the ability to sequester Nrf2 in the cytoplasm by forming the Keap1/Nrf2 complex [6]. Nrf2 is considered to act as a tumor oncogene because it is overexpressed in many cancers $[4,7,8]$. However, its role in skin cancer in vivo has been little studied. Herein, we studied the expression of Nrf2 and Keap1 proteins in normal skin tissues and malignant skin tissues in vivo.

\section{METHODS}

\section{Cell lines and tissue samples}

The human malignant melanoma cell line G361 (CRL 1424, Rockville, MD, USA) was obtained from the American Type
Culture Collection. The cells were cultured in Dulbecco's Modified Eagle's Medium (DMEM) containing 10\% fetal calf serum, $100 \mathrm{U} / \mathrm{mL}$ penicillin, $100 \mathrm{mg} / \mathrm{mL}$ streptomycin at $37^{\circ} \mathrm{C}, 5 \%$ $\mathrm{CO}_{2}$. The Institutional Review Board of Hanyang University Hospital reviewed and approved this research protocol involving the use of tissue samples. For the study, a total of 6 normal skin tissues and 18 malignant skin tumor tissues including 6 malignant melanomas (MM), 6 squamous cell carcinomas (SCC), and 6 basal cell carcinomas (BCC) were obtained from patients who underwent excisional surgery between July 2009 and December 2011 at the Department of Plastic Surgery of the Hanyang University Guri Hospital in Korea (Table 1). The specimens were immediately frozen in liquid nitrogen after excision, and stored at $-80^{\circ} \mathrm{C}$. All of the skin cancers were diagnosed by conventional pathological examination.

\section{Immunohistochemical staining}

The stored, paraffin-embedded specimens, including $6 \mathrm{MM}, 6$ SCC, and 6 BCC were used. Paraffin samples were deparaffinized in xylene, rehydrated in $10 \mathrm{mM}$ citrate buffer ( $\mathrm{pH}$ 6.0), and heated in a microwave oven for 15 minutes to restore antigens. To suppress endogenous peroxidase within the tissues, the samples were treated with $3 \%$ peroxide for 5 minutes, and then with a blocking solution for 30 minutes. Slides were incubated with the primary rabbit anti-Nrf2 antibody (SC-13032, Santa Cruz Biotechnology Inc., Santa Cruz, CA, USA) in a humid chamber for 60 minutes. Tissue staining was visualized with

Table 1. Clinical characteristics of malignant skin cancers

\begin{tabular}{|c|c|c|c|c|c|c|c|}
\hline Case & Sex/Age (yr) & Type & Site & Stage & Metastasis & Treatment & Recurrence \\
\hline 1 & Male/71 & $\mathrm{BCC}$ & Nose & T1N0M0 & - & Surgery & - \\
\hline 2 & Male/80 & $\mathrm{BCC}$ & Forehead & T1N0M0 & - & Surgery & - \\
\hline 3 & Female/91 & $\mathrm{BCC}$ & Ear & T1NOMO & - & Surgery & - \\
\hline 4 & Male/79 & $\mathrm{BCC}$ & Hand & T1NOMO & - & Surgery & - \\
\hline 5 & Female/76 & $\mathrm{BCC}$ & Nose & T1N0M0 & - & Surgery & - \\
\hline 6 & Female/60 & $\mathrm{BCC}$ & Cheek & T1NOMO & - & Surgery & - \\
\hline 7 & Male/61 & SCC & Cheek & T1N0M0 & - & Surgery & - \\
\hline 8 & Female/56 & SCC & Scalp & T2NOMO & - & Surgery & + \\
\hline 9 & Female/48 & SCC & Thigh & T3N2M1 & + & Radiation therapy & + \\
\hline 10 & Male/63 & SCC & Hand & T1N0M0 & - & Surgery & - \\
\hline 11 & Female/71 & SCC & Temple & T1N0M0 & - & Surgery & - \\
\hline 12 & Male/81 & SCC & Cheek & T1N0M0 & - & Surgery & - \\
\hline 13 & Male/60 & MM (NM) & Scalp & T3N1M1 & + & Chemotherapy & + \\
\hline 14 & Female/73 & MM (NM) & Cheek & T2NOMO & - & Surgery & - \\
\hline 15 & Male/78 & MM (ALM) & Foot & T2NOMO & - & Surgery & - \\
\hline 16 & Female/80 & MM (NM) & Cheek & T1NOMO & - & Surgery & - \\
\hline 17 & Female/76 & MM (NM) & Thigh & T2NOMO & - & Surgery & - \\
\hline 18 & Male/58 & MM (ALM) & Thumb & T1NOMO & - & Surgery & - \\
\hline
\end{tabular}


3,3'-Diaminobenzidine (ScyTek, Logan, UT, USA) substrate chromogen solution.

\section{Western blot analysis}

The human MM cell line G361 served as a positive control for Nrf2 expression. The tissue samples were homogenized in WCE buffer (25 mM HEPES [pH 7.7], 0.3 M NaCl, $1.5 \mathrm{mM} \mathrm{MgCl}$, $0.2 \mathrm{mM}$ ethylene diamine tetraacetic acid [EDTA], $0.1 \%$ Triton $\mathrm{X}-100,0.5 \mathrm{mM}$ dithiothreitol [DTT], $20 \mathrm{mM}$ glycerophosphate, $0.1 \mathrm{mM} \mathrm{Na}_{3} \mathrm{VO}_{4}, 2 \mathrm{~g}$ per mL leupeptin, $2 \mathrm{~g}$ per $\mathrm{mL}$ aprotinin, 1 $\mathrm{mM}$ phenylmethylsulfonyl fluoride [PMSF], and a protease inhibitor cocktail tablet [Boehringer Mannheim, Mannheim, Germany]). The tissue suspension was rotated at $4^{\circ} \mathrm{C}$ for $10 \mathrm{~min}$ utes. Supernatants were collected, kept at $-70^{\circ} \mathrm{C}$ and used for western blotting. Proteins from the tissue were separated by SDS-PAGE using NuPAGE 4-12\% bis-Tris gels (Invitrogen, NP0335Box) and then transferred to Immobilon-P membrane. The membrane was blocked using 5\% BSA in TBS-T $(20 \mathrm{mM}$ Tris, $\mathrm{pH}$ 7.6, $130 \mathrm{mM} \mathrm{NaCl}$, and $0.1 \%$ Tween20) solution. Then, the membrane was reacted with the primary antibody, rabbit anti-Nrf2 antibodies (SC-13032, Santa Cruz Biotechnology Inc.) diluted to $1: 1,000$ concentration at $4^{\circ} \mathrm{C}$ for 16 hours, and washed well with washing buffer and TBST buffer $(10 \mathrm{mM}$ Tris-Cl, pH 8.0, $150 \mathrm{mM} \mathrm{NaCl}, 0.05 \%$ Tween 20) 4 times for 10 minutes, 10 minutes, 15 minutes, and 15 minutes, and then reacted with anti-mouse IgG (SC-2005, Santa Cruz Biotechnology Inc.)-horseradish peroxidase-linked species-specific whole antibody diluted to 1:10,000 for 1 hour. After the reaction with antibody, the membrane was washed well 4 times for $10 \mathrm{~min}$ utes, 10 minutes, 15 minutes, and 15 minutes. Proteins on the membrane were detected using the enhanced chemiluminescence solution kit (Amersham, Bucks, UK). The membrane was stripped and reblotted with anti-actin antibody (Catalog number A5441, Sigma, St. Louis, MI, USA). For the western blot analysis of Keap1, the primary antibody used was the anti-Keap1 antibody (60027-1-Ig, Proteintech Group, Chicago, IL, USA) and the secondary antibody used was the donkey anti-goat IgGHRP (SC-2020, Santa Cruz Biotechnology Inc.).

\section{Small interfering RNA transfection}

Small interfering RNA (SiRNA) transfection was performed for detection of the accurate Nrf2 band. RNA interference of Nrf2 was performed using an Nrf2-specific siRNA duplex. Mock, control-scrambled-siRNA and Nrf2-specific siRNA were purchased from Invitrogen (Catalog number 1299001, Invitrogen, Grand Island, CA, USA). The human malignant melanoma cell line, G361, was maintained in DMEM supplemented with 5\% fetal bovine serum (FBS), $1 \mathrm{mM}$ glutamine, 100 units of peni-
cillin/mL and $100 \mu \mathrm{g}$ of streptomycin/mL. Briefly, cells were seeded in a 6-well plate and transfected at $40 \%$ confluency with siRNA duplex using lipofectamine RNAiMAX (Invitrogen) according to the manufacturer's recommendations. The adequacy of transfection was analyzed by western blot.

\section{Assessment of western blot}

The relative abundance of protein expression was analyzed using Phosphor-Imager software (TINA, from Raytest, Straubenhardt, Germany). The measured score of the expression in skin cancer tissues and normal skin tissues was compared.

\section{Statistical analysis}

The data from the Raytest TINA software were analyzed using the nonparametric Mann-Whitney U test. A P $<0.05$ was considered to be statistically significant.

\section{RESULTS}

\section{Immunohistochemical examination}

Immunohistochemical study demonstrated that the Nrf2 protein was apparently expressed in normal skin tissues. However, Nrf2 was underexpressed in BCC, SCC, and MM (Fig. 1).

\section{Western blot analysis}

Western blot analysis revealed that $\mathrm{Nrf} 2$ was definitely expressed in normal human skin tissues. In MM, SCC, and BCC tissues, Nrf2 expression was decreased significantly (Fig. 2). The Keap1 protein was not expressed in all malignant skin tumors and normal skin tissues (Fig. 3).

The relative protein expression using western blotting was plotted in a graph. The relative protein expression of Nrf2 according to the Mann-Whitney U test was analyzed. The median score of Nrf2 was found to be 0.526 (interquartile range, 0.460 0.560 ) in BCC and 0.692 (interquartile range, 0.602-0.754) in normal skin. The median score of $\mathrm{Nrf} 2$ was 0.552 (interquartile range, 0.407-0.609) in SCC and 0.853 (interquartile range, $0.781-0.867)$ in normal skin. The median score of Nrf2 was 0.665 (interquartile range, $0.566-0.695$ ) in $\mathrm{MM}$ and 0.721 (interquartile range, $0.660-0.751$ ) in normal skin (Fig. 4). There were significant differences in $\mathrm{Nrf} 2$ protein expression between malignant skin tumors and normal skin tissues $(\mathrm{P}<0.05)$.

\section{SiRNA transfection}

To confirm the accurate band level of molecular weight of Nrf2 among multiple bands in malignant skin cancers, we used the G361 cells as positive control, which were obtained after transfection with Nrf2-specific siRNA. The molecular weight of 
Fig. 1. Immunohistochemical staining for Nrf2 protein expression

Nrf2 protein was definitely expressed in normal skin but it was underexpressed in basal cell carcinoma, squamous cell carcinoma, and malignant melanoma. Brown color spots were positively stained cells. (A) Normal skin (immunohistochemical stain, $\times 100$ ). (B) Basal cell carcinoma (immunohistochemical stain, $\times 100)$. (C) Squamous cell carcinoma (immunohistochemical stain, $\times 100$ ). (D) Malignant melanoma (immunohistochemical stain, $\times 100$ ). (E) Normal skin (immunohistochemical stain, $\times 200$ ). (F) Basal cell carcinoma (immunohistochemical stain, $\times 200$ ). (G) Squamous cell carcinoma (immunohistochemical stain, $\times 200)$. (H) Malignant melanoma (immunohistochemical stain, $\times 200$ ). Nrf2, nuclear factor E2-related factor.
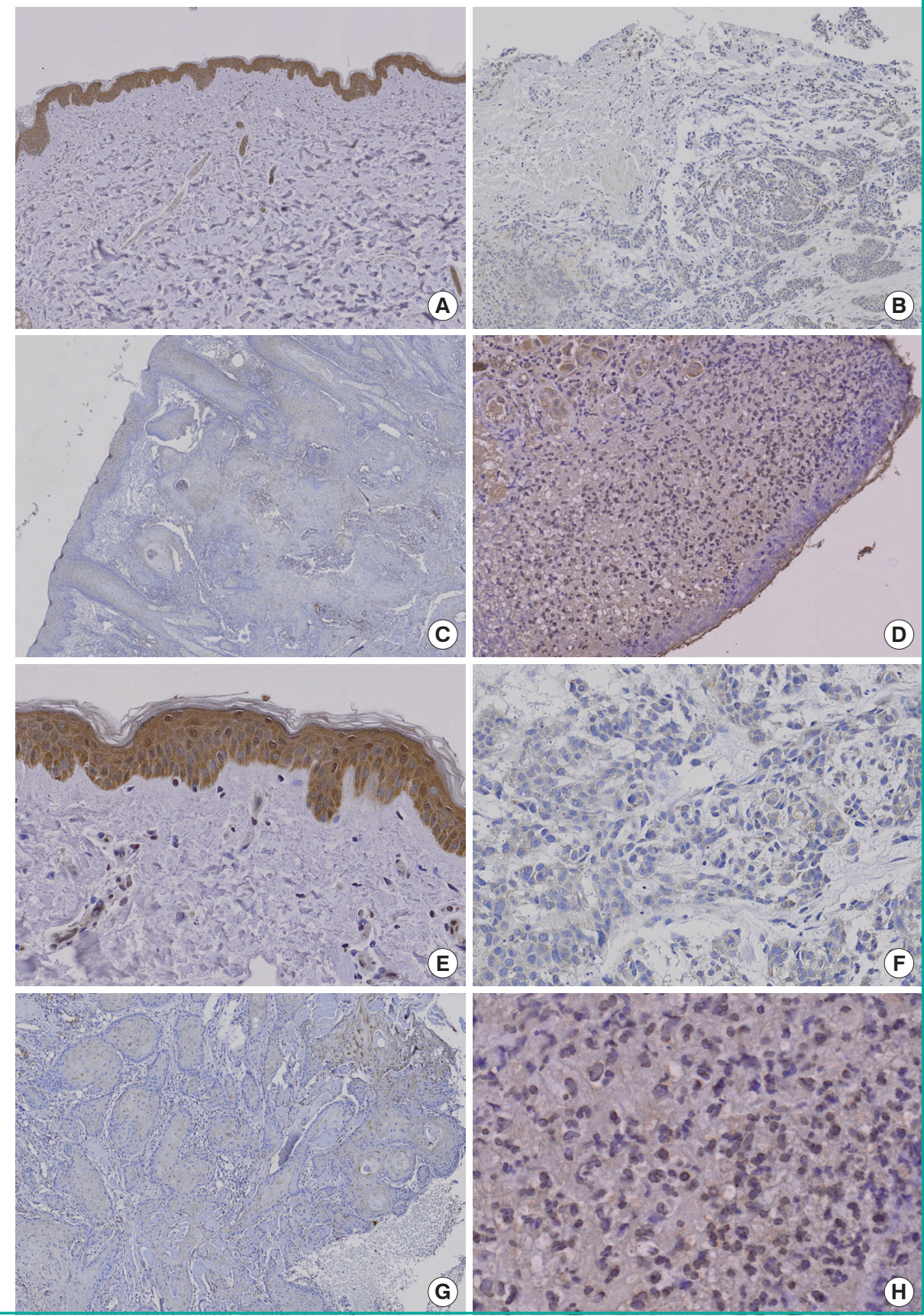

$\mathrm{Nrf} 2$ protein was analyzed as $66 \mathrm{kDa}$. Transfection of Nrf2-siRNA pointed the Nrf2 protein levels (Fig. 5).

\section{DISCUSSION}

Oxidative stress provokes cellular responses that induce the expression of genes which encode various cytoprotective proteins. Fortunately, the skin tissue has its own antioxidant systems, and one of them is Nrf2. Nrf2 maintains intracellular redox balance by controlling the transcription of target genes. Therefore, $\mathrm{Nrf} 2$ acts as a key cellular sensor for oxidative stress, and it is a critical transcriptional regulator for eliminating oxidative damage, and it protects the cells from transforming into cancer cells $[9,10]$. However, Nrf2 not only protects normal cells but also cancer cells from cellular stress, and promotes the survival of both normal and cancer cells, which indicates the dual roles of Nrf2 in both cancer prevention and promotion $[2,9,10]$.

Keap 1 is a cytoplasmic, actin-binding protein that is found to 


\section{Fig. 2. Western blot analysis of Nrf2}

Western blot showed that Nrf2 was expressed in normal human skin tissues, but Nrf2 expression was decreased in basal cell carcinoma, squamous cell carcinoma and malignant melanoma specimens. Nrf2, nuclear factor E2-related factor; BCC, basal cell carcinoma; SCC, squamous cell carcinoma.

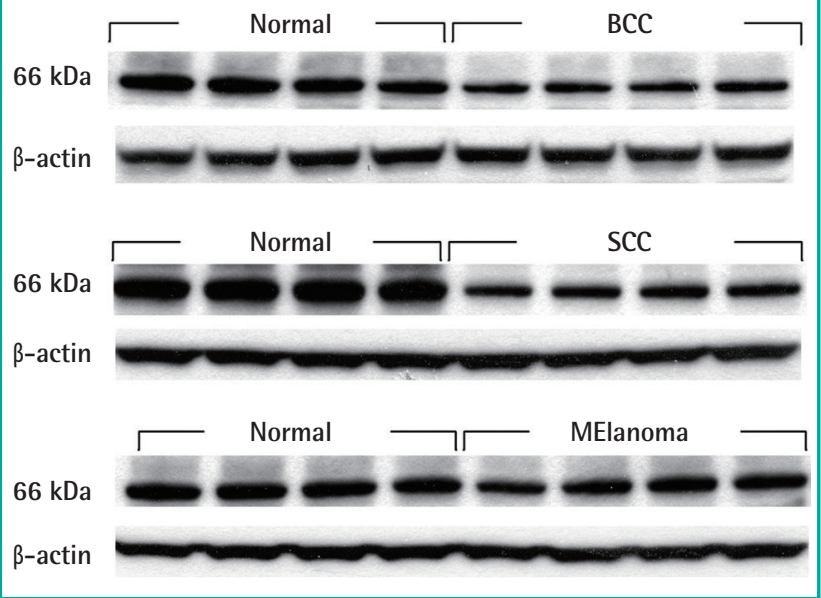

\section{Fig. 3. Western blot analysis of Keap1}

Western blot showed that the Keap1 protein was not expressed in all malignant skin tumors and normal skin tissues. Keap1, Kelch-like ECH-associated protein 1; BCC, basal cell carcinoma; SCC, squamous cell carcinoma.
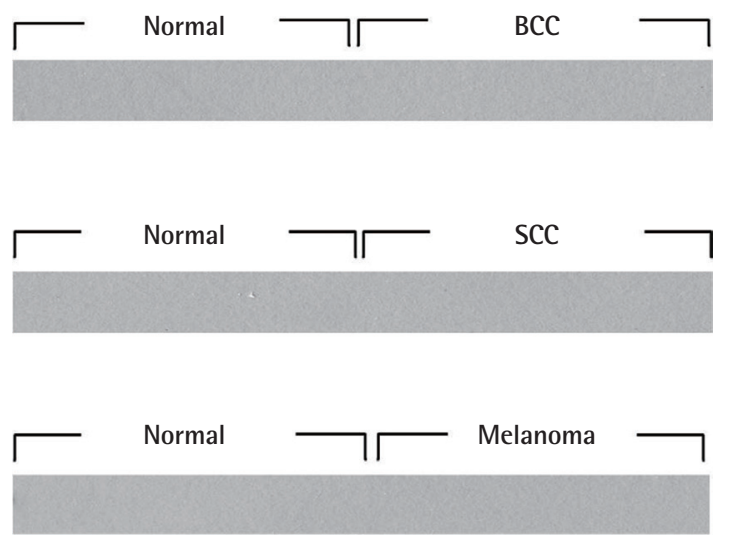

\section{Fig 4. The relative protein expression}

The relative protein expression of Nrf2 according to the MannWhitney $U$ test was analyzed. (A) The median score of Nrf2 was found to be 0.526 (interquartile range, $0.460-0.560$ ) in BCC and 0.692 (interquartile range, $0.602-0.754$ ) in normal skin. (B) The median score of Nrf2 was 0.552 (interquartile range, 0.407-0.609) in SCC and 0.853 (interquartile range, $0.781-0.867$ ) in normal skin. (C) The median score of Nrf2 was 0.665 (interquartile range, 0.5660.695 ) in $\mathrm{MM}$ and 0.721 (interquartile range, $0.660-0.751$ ) in normal skin. Nrf2, nuclear factor E2-related factor; BCC, basal cell carcinoma; SCC, squamous cell carcinoma; NM, nodular melanoma.
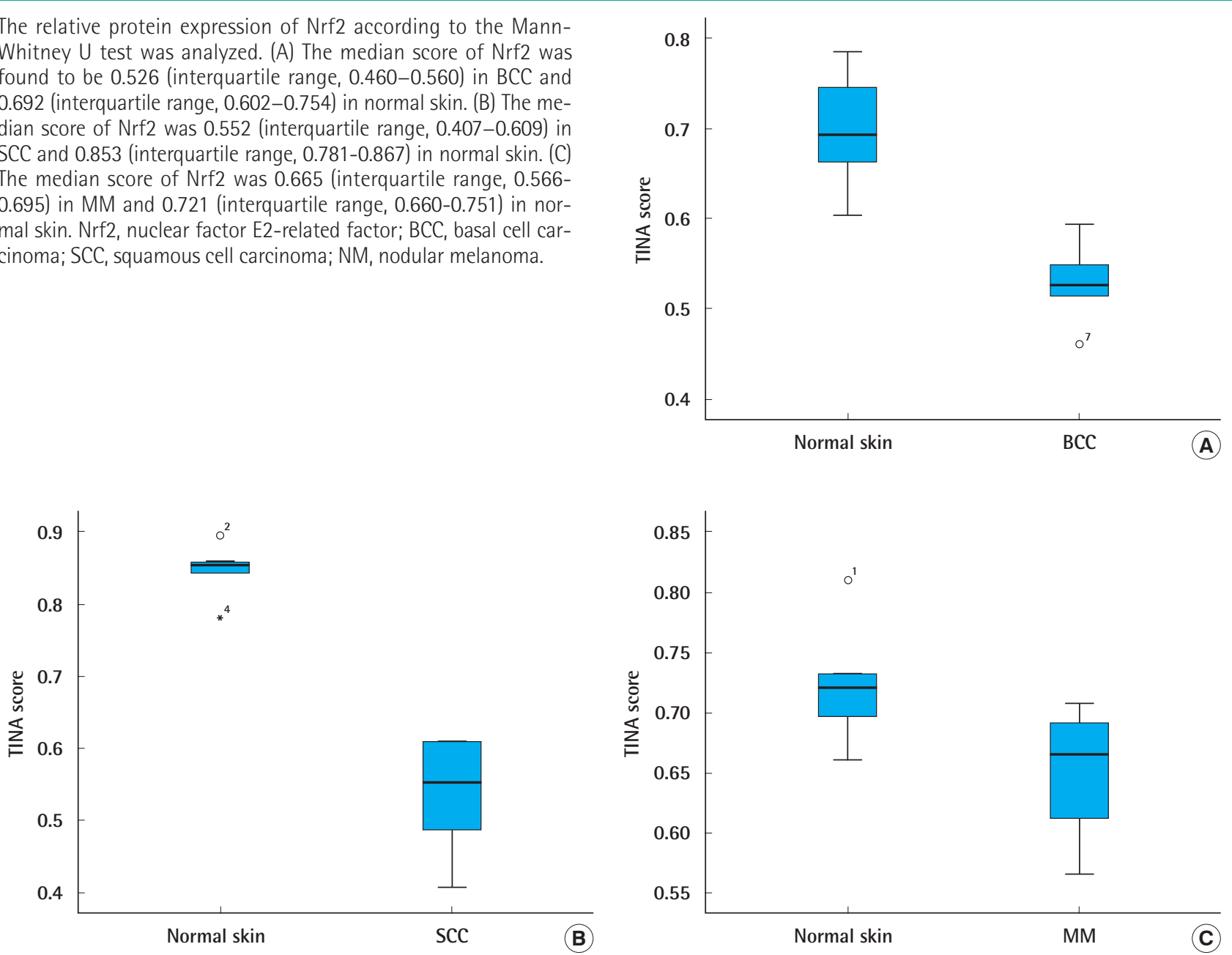

0.4

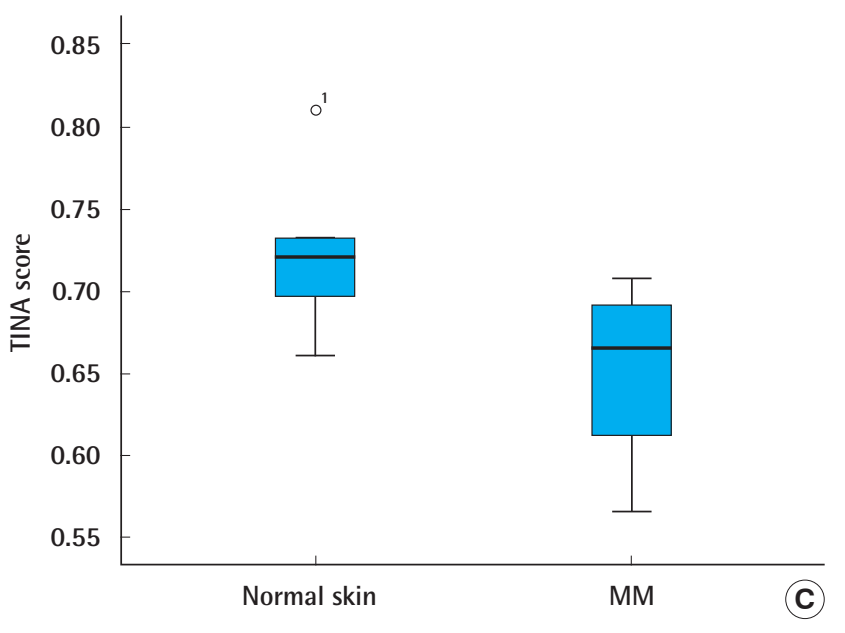




\section{Fig. 5. Small interfering RNA against Nrf2}

The exact molecular weight of Nrf2 was confirmed by mock, control scrambled siRNA and Nrf2-siRNA transfection in the G361cell line. Black arrow was absent compared with siRNA control and G361 siRNA transfection. Nrf2, nuclear factor E2-related factor; siRNA, small interfering RNA.

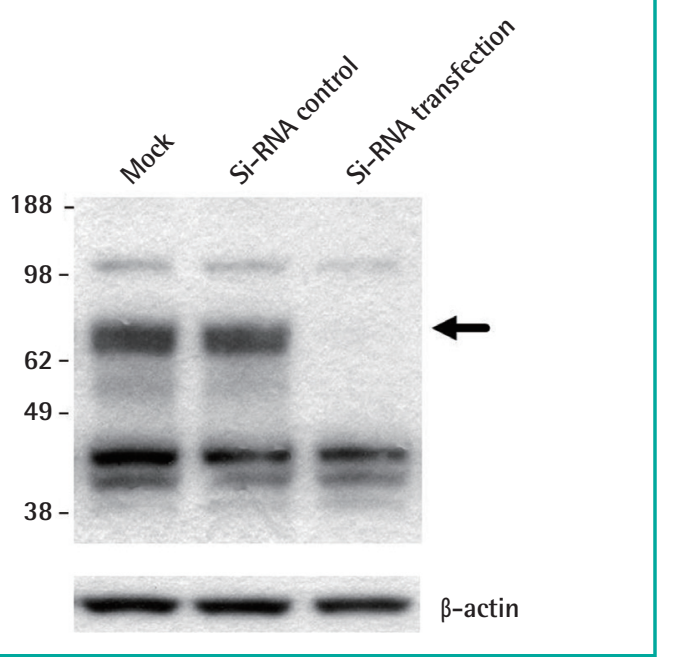

be a potent cytosolic repressor of $\mathrm{Nrf} 2$ [6]. As a key regulator of Nrf2, Keap1 sequesters Nrf2 in the cytoplasm by forming the $\mathrm{Nrf2/Keap} 1$ complex, preventing its entry into the nucleus under normal conditions. Previously, Keap1 was known as the primary redox sensor [11]. The redox signal encoded by Keap1 is subsequently transmitted to $\mathrm{Nrf} 2$, which causes the release of Nrf2 from Keap1. However, recent studies have reported that the relay of the redox signal from Keap1 to Nrf2 may not happen in vivo. Studies reported the dysfunction of Keap1 in lung cancer and gallbladder cancer, which elevated the levels of Nrf2 $[12,13]$. Recently, Bryan et al. reported that Nrf2 translocated into the nucleus via the Keap 1 dependent or independent pathway [5]. In fact, our study demonstrated that Keap1 was not expressed in skin cancers as well as in normal skin in vivo. This finding suggests that the Nrf2/Keap1 system may be dysregulated in human skin cancers, and Nrf2 is translocated into the nucleus via the independent pathway in skin cancers.

$\mathrm{Nrf2}$ has been reported to be upregulated in various types of cancers, including primary head and neck cancers [8], lung [12], gallbladder [13], liver [14], stomach [15], breast [16], and endometrial cancers [17], and this indicates an oncogenic role of Nrf2 in cancers. There are a few reports on skin cancers. Pi et al. [18] showed that Nrf2 was involved in arsenic-induced malignant transformation of human keratinocytes. Kim et al. [9] reported that enhanced Nrf2 expression was observed in cutaneous SCCs, and Nrf2 act as an oncogene in SCC. They analyzed the Nrf2 protein expression only by immunohistochemistry. However, in our study, Nrf2 was under-expressed in cutane- ous SCCs. In contrast to the study by Kim et al. [9], we evaluated the Nrf2 protein level by immunohistochemical examination and verified it by Western blot analysis. In our study, Nrf2 expression was definitely detected in normal skin tissue, but expression of Nrf2 was definitely decreased in malignant skin cancers. Kwak et al. [19] showed that Nrf2-knockout mice were more prone to tumor formation when they were exposed to carcinogens. Xu et al. [20] reported that Nrf2-null mice developed significantly more number of skin tumors than did wild-type mice. In our study, decreased expression of $\mathrm{Nrf} 2$ may be related to skin carcinogenesis because $\mathrm{Nrf2}$ was identified as a principal regulator of oxidant defense that is related to cancer formation.

ROS play a pivotal role in survival of all living organisms, because dysregulated ROS can lead to molecular damage, DNA mutation, apoptosis, cell transformation, and cancer. Many studies on oxidative stress and carcinogenesis have been reported $[21,22]$. Among them, various antioxidant proteins are highly expressed or underexpressed. Therefore, the protective mechanisms against ROS are vital for cell survival, and one of the protective systems is Nrf2. The main biologic function of $\mathrm{Nrf} 2$ is antioxidant defense [23]. We believe that down-regulation of Nrf2 is related to skin cancers. We also demonstrated that skin cancers and normal skin tissues did not express the Keap 1 protein. Further studies are needed to better understand the mechanism of signal transduction and regulation of Nrf2 in skin cancers.

\section{REFERENCES}

1. Bickers DR, Athar M. Oxidative stress in the pathogenesis of skin disease. J Invest Dermatol 2006;126:2565-75.

2. Breimer LH. Molecular mechanisms of oxygen radical carcinogenesis and mutagenesis: the role of DNA base damage. Mol Carcinog 1990;3:188-97.

3. Rosen GM, Pou S, Ramos CL, et al. Free radicals and phagocytic cells. FASEB J 1995;9:200-9.

4. Marrot L, Jones C, Perez P, et al. The significance of Nrf2 pathway in (photo)-oxidative stress response in melanocytes and keratinocytes of the human epidermis. Pigment Cell Melanoma Res 2008;21:79-88.

5. Bryan HK, Olayanju A, Goldring CE, et al. The Nrf2 cell defence pathway: Keap1-dependent and -independent mechanisms of regulation. Biochem Pharmacol 2013;85: 705-17.

6. Kobayashi M, Itoh K, Suzuki T, et al. Identification of the interactive interface and phylogenic conservation of the Nrf2Keap1 system. Genes Cells 2002;7:807-20.

7. Arlt A, Bauer I, Schafmayer C, et al. Increased proteasome 
subunit protein expression and proteasome activity in colon cancer relate to an enhanced activation of nuclear factor E2related factor 2 (Nrf2). Oncogene 2009;28:3983-96.

8. Stacy DR, Ely K, Massion PP, et al. Increased expression of nuclear factor E2 p45-related factor 2 (NRF2) in head and neck squamous cell carcinomas. Head Neck 2006;28:813-8.

9. Kim YR, Oh JE, Kim MS, et al. Oncogenic NRF2 mutations in squamous cell carcinomas of oesophagus and skin. J Pathol 2010;220:446-51.

10. Motohashi H, Yamamoto M. Nrf2-Keap1 defines a physiologically important stress response mechanism. Trends Mol Med 2004;10:549-57.

11. Wakabayashi N, Dinkova-Kostova AT, Holtzclaw WD, et al. Protection against electrophile and oxidant stress by induction of the phase 2 response: fate of cysteines of the Keap 1 sensor modified by inducers. Proc Natl Acad Sci U S A 2004; 101:2040-5.

12. Singh A, Misra V, Thimmulappa RK, et al. Dysfunctional KEAP1-NRF2 interaction in non-small-cell lung cancer. PLoS Med 2006;3:e420.

13. Shibata T, Kokubu A, Gotoh M, et al. Genetic alteration of Keap1 confers constitutive Nrf2 activation and resistance to chemotherapy in gallbladder cancer. Gastroenterology 2008; 135:1358-68.e1-4.

14. Ikeda H, Nishi S, Sakai M. Transcription factor Nrf2/MafK regulates rat placental glutathione S-transferase gene during hepatocarcinogenesis. Biochem J 2004;380:515-21.

15. Wang HB, Zhou CJ, Song SZ, et al. Evaluation of Nrf2 and IGF-1 expression in benign, premalignant and malignant gastric lesions. Pathol Res Pract 2011;207:169-73.

16. Nioi P, Nguyen T. A mutation of Keap1 found in breast cancer impairs its ability to repress Nrf2 activity. Biochem Biophys Res Commun 2007;362:816-21.

17. Jiang T, Chen N, Zhao F, et al. High levels of Nrf2 determine chemoresistance in type II endometrial cancer. Cancer Res 2010;70:5486-96.

18. Pi J, Diwan BA, Sun Y, et al. Arsenic-induced malignant transformation of human keratinocytes: involvement of Nrf2. Free Radic Biol Med 2008;45:651-8.

19. Kwak MK, Wakabayashi N, Itoh K, et al. Modulation of gene expression by cancer chemopreventive dithiolethiones through the Keap1-Nrf2 pathway. Identification of novel gene clusters for cell survival. J Biol Chem 2003;278:813545.

20. Xu C, Huang MT, Shen G, et al. Inhibition of 7,12-dimethylbenz(a)anthracene-induced skin tumorigenesis in C57 $\mathrm{BL} / 6$ mice by sulforaphane is mediated by nuclear factor E2-related factor 2. Cancer Res 2006;66:8293-6.

21. Dairkee SH, Nicolau M, Sayeed A, et al. Oxidative stress pathways highlighted in tumor cell immortalization: association with breast cancer outcome. Oncogene 2007;26:626979.

22. Reuter S, Gupta SC, Chaturvedi MM, et al. Oxidative stress, inflammation, and cancer: how are they linked? Free Radic Biol Med 2010;49:1603-16.

23. Li W, Kong AN. Molecular mechanisms of Nrf2-mediated antioxidant response. Mol Carcinog 2009;48:91-104. 